\title{
Meter Reading Application for Android Based Handheld Device
}

\author{
Mayur Suryawanshi ${ }^{1}$, Aditya Nikam ${ }^{2}$, AjayKumar Gajengi $^{3}$, Dakshata Waje $^{4}$ \\ Scholar, Department of Information Technology, ISB\&M SOT, Pune, India ${ }^{1,2,3,4}$
}

\begin{abstract}
Automatic meter reading is an Application designed for collecting consumption automatically, and status data from utility meters (electricity) and transferring data to a central database for billing. This is an application which will help you to monitor your consumption of e.g. electricity. You may have savings in your budget if you track your monthly consumption of energy. The Application is built essentially to track the energy usage and accessing the electricity data which can result in better management electricity. Enabling accurate data stored, analysed and provided to a customer on demand. This Application is used in utility meters for collecting the data which is needed for billing purposes.
\end{abstract}

Keywords: Android, OCR, Handheld device, Android based meter reader.

\section{INTRODUCTION}

Now a days most of utility service provider companies use • Completely manual process.

manual process for taking readings. Those readings are $\bullet$ Increase burden on meter reader.

maintained in record book. Due to manual processing of $\bullet$ More possibilities of human errors.

data there are possibilities of generating an incorrect bill. • Time consuming.

This process cause extra work load on person who is capturing meter readings and there is no such way by which customer can easily report a complaint or file complaint, if any problem occurs. To solve this problem we are proposed an android [6] application which will carry by meter reader to take readings and web portal for electricity users to interact with electricity Distribution Company. Meter reader simply captures image of meter, then application performs OCR operation (extraction of text from image) and send captured readings to server.

The sever will generate bill according to customers meter reading, after updating database, PDF is send to customer via registered email address. Thus this process reduces manual work, so wrongness in the bill generation is decreased. Thus, that time consuming process is turned into completely accurate automated process.

\section{EXISTING SYSTEM}

The existing system is an electricity distribution company which gives contract to collect meter reading of customer's electricity reading. Then the worker of third Part Company takes reading and submits it to the administrator office. Administrator office sends that data to electricity distribution system. Electricity Distribution Company delivers the generated bill to the customers according to their meter reading. The companies who used to perform manual work for this process assumes that the process of bill generation is easy and they do not require skilled worker so they don't need invest on adapting new technology for billing. In case of incorrect bill and device failure customer has to face difficulties for lodging complains. There is no communication channel provided by company for broadcasting different information such as power failure.

Problems in Existing System: -

\section{III.PROPOSE SYSTEM}

We are developing the Solution for meter reader and a web portal for customer. By using Android application, reader will get the meter reading. Meter reader carries handheld device [1] with android Operating system in which our android application is installed.

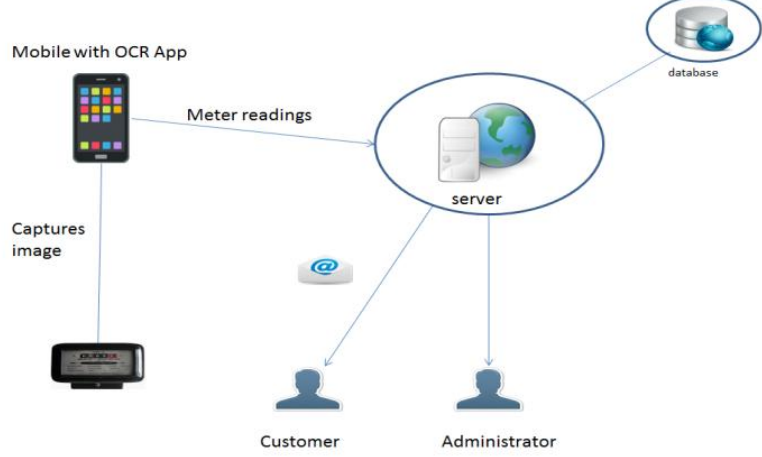

Fig1.System architecture of proposed system

This process of bill generation is very simple. Meter reader needs to capture image of meter, then the application perform OCR operation on captured image, outcome of OCR operation i.e. reading are send to server for bill calculation and bill generation process.

The sever will generate bill according to customers meter reading, after updating database PDF is send to customer via registered email address.

\section{IV.MATHEMATICAL MODEL}

1. LET SYSTEM AS $S=\{\ldots\}$

2. IDENTIFY INPUT AS INP, THEN $S=\{$ INP... $\}$

INP1: METER READER OR USER LOGIN.

INP2: CAPTURED IMAGE OF METER. 


\section{IDENTIFY OUTPUT OPT}

$\mathrm{S}=\{\mathrm{INP}, \mathrm{OPT} \ldots\}$

OPT1: GENERATED BILL.

OPT2: SEND PDF TO CUSTOMER.

4. IDENTIFY THE PROCESS AS P

$\mathrm{S}=\{$ INP, OPT, PROC $\ldots\}$

PROC0: GREY-SCALE CONVERSION.

PROC1: THRESHOLDING.

PROC2: OCR OPERATION.

PROC3: BILl GENERATION.

PROC4: SEND PDF

5. IDENTIFY SUCCESS AS SUCC.

$\mathrm{S}=\{$ INP, OPT, PROC, SUCC.... $\}$

SUCC $=\{$ SUCCESS OCCURS, IF BILL IS GENERATED ACCURATELY. $\}$

6. IDENTIFY FAILURE AS FAIL.

$\mathrm{S}=\{$ INP, OPT, PROC, SUCC, FAIL ... $\}$

FAIL $=\{$ FAILURE OCCURS IF CASE OF INCORRECT BILL GENERATION.\}

7. CLASSIFY INITIAL CONDITION AS INICON.

$\mathrm{S}=\{\mathrm{INP}$, OPT, PROC, SUCC, FAIL, INICON $\}$

INICON $=\{$ METER IMAGE IS COMPULSORY. $\}$

\section{CONCEPT AND TECHNOLOGIES}

\section{A. OCR (Optical character recognition)}

OCR [2] is a system that provides a full alphanumeric recognition of printed or handwritten characters at electronic speed by simply scanning the document.

There are 3 phases in OCR:

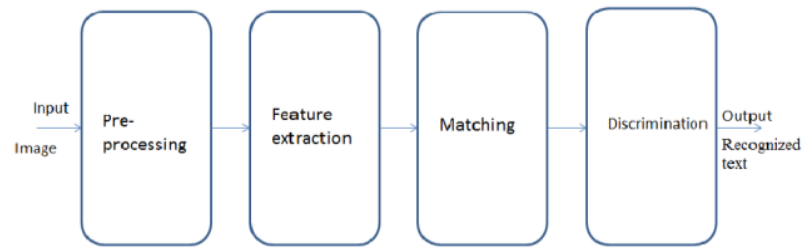

Fig2.System architecture of proposed system

\section{Pre-processing}

Pre-processing produces data for OCR to operate accurately. Pre-processing improves recognition rate.

It consists of following techniques:

1.1De-skew

If the document is not aligned perfectly then it may need to tilt in order to make text perfectly horizontal or vertical.

\subsection{Binarization}

It is the process of converting a pixel image into a binary image.

The Otsu [3] Thresholding method is used for binarization process.

a) Compute histogram and probabilities of each intensity level

b) Set up initial $\omega i(0)$ and $\mu(0)$

c) Step through all possible thresholds $t=1 \ldots$ maximum intensity i) Update $\omega \mathrm{i}$ and $\mu \mathrm{i}$

ii) Compute $\sigma_{b}^{2}(t)$

d) Desired threshold corresponds to the maximum $\sigma_{b}^{2}(t)$

\section{Feature extractions:}

It scans and selects the set of features that classifies and analyse the character. This phase will maximize the recognition rate.

3. Recognition:

Recognition process divided into two sub processes Matching and Discrimination.

3.1 Matching

In matching phase, features that extracted from image, are matched with Template of character.

3.2 Discrimination

In discrimination feature that are matched, are converted into character. The matched features are converted into character code.

\section{B. ANDROID}

Now days android [6] is most popular operating system for touch screen devices like smartphones, tablet computers as well and other many devices. Android uses Linux kernel. User interface of android OS is based on direct manipulation by using touch as inputs that are loosely correspond to real word action as tapping swiping and pinching to perform various activities with on screen objects.

Android is basically designed to keep consumption of low power, for memory management of handheld device. The memory management is done by suspending android application which is no longer in use. Suspended application is still technically open, which consume no resource and idly sit in the background unless and until needed again

We selected this technology for our project because, now a day's most of smart phones are running on android, and it is simple to user.

\section{C. $P H P$}

Php is a server side scripting programming language used for web development. But it can be used as general purpose programming language. $\mathrm{Php}$ is free software which is released under the PHP license.it can be deploying on every operating system platform.

PHP code may be embedding into HTML or it can combine with different web frameworks and web template system. PHP code is usually handled by a PHP interpreter [4] implemented as a module in the web server which generates result as webpage.

We can execute PHP code by using command line interface (CLI).In PHP5 gives features like concept of object oriented programming, and performance enhancements.

Now a days PHP is installed on more than 2.1 million web servers and over 244 million websites. 


\section{D. $M y S Q L$}

MySQL [8] is most popular open source database server. SQL is abbreviate ion of "structured query language", which is to interact with database by accessing information and updating information from database.

Various queries can be performed like data selection, insertion, updating, and locating. Basically database is collection for well-organized information that allow for easy retrieval. MySQL offer two editions a) Community Edition (open source) and 2) a Network Edition (licensed).

\section{E. TESSERACT}

Tesseract [5] is an OCR engine for various operating systems. It is released under Apache license, v2.it is free software, development of Tesseract has been sponsored by google.it is considered to one of the most accurate OCR engine currently available.

\section{F. $D O M P D F$}

DOMPDF [7] is library, which is capable of generating pdf from HTML mark-up and CSS style. It gives flexibility to decide how our content should look using familiar technology and convert them into pdf. This library offers other useful and interesting features too.

\section{VI.CONCLUSION}

Meter reading application for android based handheld device suggests the solutions to address problem related to various utility meters.

The current technology uses process which is totally dependent on manual process such as meter reading, entering reading manually to server and generating and distributing bill to customers. Many customers complain about incorrectness of bill, this is because of improper meter reading and lead to major disadvantage of current technology.

Our solution is implemented on meter reader so that his workload is being reduced. As our solution sends reading to server after capturing image, this will help to reduce human error as well as to automate and accurate process.

For future development, we suggest to send utility bill by Short messaging service (SMS) to customer who does not have email address

\section{ACKNOWLEDGMENT}

The authors would like to thank Department of Information Technology, IS\&M SOT and indebted to our guide and co-guide for their guidance and sagacity without which this paper would not have been designed. He provided us with valuable advice which helped us to accomplish the design of this paper. We are also thankful to our HOD (Department of Information Technology) for her constant encouragement and moral support. Also we would like to appreciate the support and encouragement of our colleagues who helped us in correcting our mistakes and proceeding further to produce the paper with the required standards.

\section{REFERENCES}

[1] Design of an Optical Character Recognition System for Camera based Handheld Devices -IJCSI International Journal of Computer Science Issues, Vol. 8, Issue 4, No 1, July 2011

[2] "A Review on the Various Techniques used for Optical Character Recognition", Pranob K Charles, V.Harish, M.Swathi, CH. Deepthi/ International Journal of Engineering Research and Applications (IJERA) ISSN: 2248-9622, Vol. 2, Issue 1,Jan-Feb 2012.

[3] www.wseas.us/e-library/conferences/2013/Antalya/ITCN/ITCN11.pdf

[4] https://en.wikipedia.org/wiki/PHP_interpreter

[5] The Tesseract open source OCR engine,http://code.google.com/p/tesseract-ocr.

[6] https://en.wikipedia.org/wiki/Android_(oprating_system)

[7] http://github.com/dompdf

[8] https://en.wikipedia.org/wiki/MySQL

\section{BIOGRAPHIES}

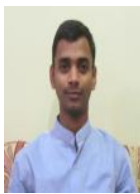

Mayur Vishawanath Suryawanshi is currently pursuing Bachelor of Engineering in Information technology in ISB\&M School of Technology, Pune affiliated to Savitribai Phule Pune University

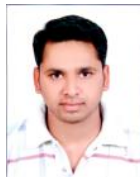

Aditya Prabhakar Nikam is currently pursuing Bachelor of Engineering in Information technology in ISB\&M School of Technology, Pune affiliated to Savitribai Phule Pune University

AjayKumar Gajengi is currently pursuing Bachelor of Engineering in Information technology in ISB\&M School of Technology, Pune affiliated to Savitribai Phule Pune University

Dakshata Dinanath Waje is currently pursuing Bachelor of Engineering in Information technology in ISB\&M School of Technology, Pune affiliated to Savitribai Phule Pune University 